\title{
Le changement d'un seul acide aminé peut altérer la spécificité d'une enzyme (cytochrome P 450)
}

Malgré les progrès réalisés dans la connaissance de la structure des protéines, ce qui fait la spécificité des enzymes reste difficile à comprendre. Qu'une mutation unique puisse abolir une activité enzymatique est bien connu en pathologie. Un changement de spécificité, en revanche, s'observe rarement, et on se souvient du cas, déjà relaté par $\mathrm{m} / \mathrm{s}\left(n^{\circ} 2\right.$, vol. 1 , p. 106), où le remplacement d'une méthionine par une arginine a transformé, chez un malade, une activité antitrypsine en antithrombine. Des expériences de mutagenèse dirigée ont permis à Lindberg et Negishi (Caroline du Nord, USA) de montrer l'effet décisif de certaines substitutions ponctuelles d'acides aminés. Ils ont choisi comme modèles [1] deux molécules de cytochrome P450 de souris. Les cytochromes P450 mitochondriaux ont une spécificité de substrat très étroite, contrairement à ceux qui sont localisés dans les microsomes. Les deux composés sont le cytochrome $\mathrm{P} 450_{\text {oh }}$, qui hydroxyle la coumarine en 7-hydroxycoumarine, et le $\mathrm{P}^{4} 50_{15 \sigma}$, qui transforme la testostérone en 15-hydroxytestostérone. Leur spécificité est très précise, bien que leurs séquences soient très voisines: sur les 494 acides aminés qu'ils comptent tous deux, 11 seulement sont différents. Les auteurs ont entrepris de remplacer chacun des 11 acides aminés d'un P450 par celui qui est présent dans l'autre par mutagenèse dirigée. Les mutants - ainsi que les types sauvages - ont été transférés dans des cellules COSI, et les deux activités hydroxylase ont été mesurées dans les cellules. En partant du $\mathrm{P} 450_{\mathrm{coh}}$, trois des 11 acides

pour l'activité coumarine hydroxylase (Tableau I). Chacune des trois mutations fait tomber l'activité de plus de moitié, et celle-ci disparaît complètement si l'on mute les trois simultanément, alors que la protéine reste à un niveau normal comme en témoigne le dosage immunologique. Une mutation unique (Phe $209 \rightarrow$ Leu) confère à la molécule une forte activité vis-à-vis de la testostérone. La mutation inverse à partir du

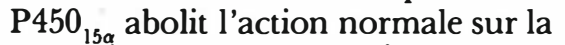
testostérone, montrant l'importance décisive de cet acide aminé. Une des mutations (Ala $117 \rightarrow$ Val) provoque l'apparition de l'action sur la coumarine, totalement absente du type normal.

L'effet de mutations ponctuelles sur la spécificité de réactions enzymatiques, semblables (hydroxylation), mais s'adressant à des substrats très différents, est donc frappant. Est-il possible d'en tirer des conclusions sur le mode de fonctionnement de ces enzymes? Le fait que trois acides aminés, placés très à distance sur la séquence, interviennent, fait penser qu'ils doivent se rapprocher dans l'espace ; l'étude des inhibitions réciproques de deux activités a fait conclure que les sites catalytiques des deux réactions étaient voisins mais distincts. La principale difficulté vient de ce qu'on ne connaît encore aucune structure d'un cristal de P450 provenant d'un mammifère, bien que plus de 60 séquences soient publiées. La seule référence est celle de la structure cristalline du P450 cam de Pseudomonas putida, dont le substrat est le camphre [2]. Cependant, les tentatives d'alignement afin d'élucider la structure dans l'espace des $\mathrm{P} 450$ de mammifères se heurtent à un problème délicat [3] : bien que le cytrochrome de Pseudomonas soit moins long (il n'a que 414 acides aminés), on peut voir que le résidu 209, le plus important pour la fonction, est très loin de la poche

Tableau I

ACTIVITÉS ENZYMATIQUES DES DEUX CYTOCHROMES P450 (en picomoles par minute par $\mathrm{mg}$ de protéine),

\begin{tabular}{|llll|}
\hline $\begin{array}{l}\text { Composé de } \\
\text { départ }\end{array}$ & Acide aminé modifié & coh & $\mathbf{1 5} \alpha$ \\
\hline P450 $_{\text {coh }}$ & non modifié & 20,4 & 0,12 \\
& Val 117 $\rightarrow$ Ala & 7,4 & 0,25 \\
& Phe 209 $\rightarrow$ Leu & 8,8 & 11,50 \\
& Met 365 - Leu & 9,8 & 0,12 \\
& les trois mutations ensemble & 0,0 & 14,10 \\
\hline P450 $_{15 \alpha}$ & non modifié & 0,0 & 15,0 \\
& Ala 117 $\rightarrow$ Val & 5,1 & 30,1 \\
& Leu 209 $\rightarrow$ Phe & 1,8 & 0,12 \\
& Leu 365 $\rightarrow$ Met & 1,3 & 49,8 \\
\hline
\end{tabular}


de l'hème, ce qui fait invoquer un effet à distance, toujours difficile à démontrer. On voit donc que bien du chemin reste à parcourir avant de comprendre parfaitement les mécanismes qui gouvernent la spécificité des enzymes.

J.C.D

1. Lindberg RLP, Negishi M. Alteration of mouse cytochrome $\mathrm{P} 450$ coh substrate specificity by mutation of a single aninoacid residue. Nature 1989 ; 339 : 632-4.

2. Poulos TL, Finzel BC, Howard AJ. High resolution crystal structure of cytrochrome

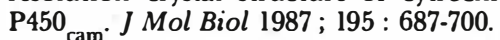

3. Poulos TL. Reversing enzyme specificity. Nature $1989 ; 339$ : 580-1.

\section{BRĖVES}

Les anticorps anti-HIV à effet facilitant : infection indépendante de CD4, utilisant le récepteur Fc. Il existe des anticorps anti-HIV qui, loin de protéger contre l'infection virale, peuvent au contraire faciliter cette infection, ce qui pose le très difficile problème que l'on peut supposer concernant le développement d'une vaccination dont on espère au moins qu'elle ne risque jamais d'être aggravante! Le groupe de J.A. Levy (San Francisco, CA, USA) vient d'établir que les anticorps facilitants agissaient en permettant probablement l'infection de cellules n'exprimant pas la protéine $\mathrm{CD} 4$, récepteur habituel du virus. L'anticorps, fixé au virus par son site spécifique d'un épitope antigénique, peut se lier au récepteur pour le fragment Fc (fragment cristallisable, dans la portion constante des chaînes lourdes des immunoglobulines) exprimé sur certaines cellules. Cette étude a été faite seulement avec des macrophages et des lymphocytes $T$, et des données ultérieures sont nécessaires pour déterminer quel est le caractère de généralité du phénomène.

[Homsy J, et al. Science 1989; 244 : 1357-60.]

$m / s n^{\circ} 7$ vol. 5 , septembre 89

\section{BRÈVES}

Seuls les 106 premiers acides aminés de la protéine CD4 sont indispensables à la liaison du virus HIV à la glycoprotéine gpl20. Parmi les démarches à visée thérapeutique destinées à combattre le SIDA, celles fondées sur l'utilisation de molécules CD4 solubles sont peut-être prometteuses ( $m / s n^{\circ} 4$, vol. 5 , p. 266). Rappelons que la molécule membranaire CD4 constitue le récepteur principal du virus HIV, permettant notamment sa fixation sur les lymphocytes T CD4 ${ }^{+}$. CD4 est une protéine qui appartient à la «superfamille » de type immunoglobuline, avec notamment quatre domaines extracellulaires ressemblant à des segments $\mathrm{V}$ J de molécules d'anticorps (domaines Vla, V4). Un travail coopératif entre les laboratoires américains, anglais et italiens vient de confirmer et de préciser des résultats déjà évoqués par d'autres groupes : l'interaction entre le virus et CD4 se fait par l'intermédiaire de la région VlM [1]. Une protéine tronquée ne possédant que ce domaine se lie à gpl20 de HIV avec une forte affinité et inhibe ex vivo l'infection de lymphocytes $\mathrm{T}$ par le virus. Le site d'interaction entre gpl 20 et CD4 a même été plus précisément identifié comme étant un court segment de 12 résidus. Toutes ces données seront importantes pour la conception de nouveaux agents thérapeutiques potentiels fondés sur l'inhibition de la liaison du virus HIV à son récepteur CD4.

[1. Arthos J, et al. Cell 1989; 57 : 469-81.]

Une demande de diagnostic prénatal à motivation inhabituelle. L'indication essentielle du diagnostic prénatal est le risque que court le foetus d'être atteint d'une affection grave, qu'elle soit héréditaire, congénitale ou acquise. Mais parfois une demande émanant de la famille pose des problèmes éthiques redoutables. La plus fréquente est une demande de diagnostic précoce de sexe que motive non pas le risque d'une maladie liée au chromosome $\mathrm{X}$, mais le désir des parents de s'assurer un enfant de sexe défini en éliminant le fotus du sexe non désiré. Jusqu'à présent, en France du moins, il a été possible de résister à ce type de pression, à la fois pour des raisons éthiques et parce que les possibilités techniques sont déjà saturées par les demandes médicalement justifiées. Le problème qu'ont eu à affronter Clark et al. (universités de CA et VA, USA) est d'une autre nature [1]. Une femme - non enceinte - de 27 ans avait un garçon de deux ans atteint de syndrome de Wiscott-Aldrich. Un de ses frères était mort dans l'enfance de cette maladie redoutable, qui associe troubles cutanés, digestifs et sanguins, et qui peut faire l'objet d'un diagnostic prénatal depuis qu'on a $\mathrm{pu}$ localiser son gène avec précision sur le chromosome X [2]. La mère avait eu connaissance du succès d'une greffe de moelle osseuse provenant d'un membre sain de la fratrie et de même groupe HLA. Ce qu'elle demandait n'était pas un diagnostic de Wiscott-Aldrich, mais un typage HLA chez le fœetus. Le couple se déclarait prêt à subir autant d'avortements qu'il le faudrait jusqu'à l'obtention du fœetus désiré, exempt bien entendu de la maladie, mais surtout de groupe HLA identique à celui de l'enfant atteint. Ce qu'il y a donc de particulier dans ce cas est que l'enfant n'était pas désiré pour luimême, mais seulement à titre de donneur de greffe pour son frère aîné. Après bien des hésitations, les auteurs ont décidé de ne pas se prêter à cette opération. Un tel exemple permet de prévoir que des situations difficiles apparaîtront encore dans l'avenir.

[1. Clark RD, et al. Prenatal Diagn $1989 ; 9$ : 329-34.]

[2. Peacocke M, Simonovitch KA. Proc Natl Acad Sci USA 1987 ; 84 : 3430-3.] 
Cascade d'activations d'oncogènes nucléaires. L'adénovirus de type 5 contient deux oncogènes d'expression ultraprécoce au cours du cycle viral, ElA et ElB. ElA est une protéine nucléaire susceptible d'activer en trans d'autres gènes, viraux ou cellulaires $\left(\mathrm{m} / \mathrm{s} n^{\circ} 7\right.$, vol. 2, p. 410), mais qui ne semble pas capable de se lier, seule et par elle-même, à des séquences spécifiques d'ADN. Elle agit plutôt en coopération avec d'autres protéines qu'elle pourrait activer (par exemple par phosphorylation) ou auxquelles elle pourrait se complexer. C'est ainsi que ElA active un facteur transcriptionnel protéique ubiquitaire dénommé E2F qui se comporte lui-même comme une protéine se liant à des motifs spécifiques d'ADN (comportant la séquence 5'GCGCGAAA-3') et activant des promoteurs et enhancers de gènes d'expression précoce d'adénovirus. Deux équipes [1, 2] viennent de démontrer que ce même facteur E2F pouvait stimuler la transcription de l'oncogène $c-m y c$ en se fixant au niveau de l'un de ses deux promoteurs alternatifs proximaux, $\mathrm{P}_{2}$ (il existe aussi un promoteur distal, $\mathrm{P}_{0}, \mathrm{~m} / \mathrm{s} n^{\circ} 3$, vol. 3 , p.170). E2F peut lui-même être activé non seulement par $\mathrm{ElA}$, mais aussi par des substances cellulaires de fonction équivalente dont l'existence est suspectée depuis longtemps $(\mathrm{m} / \mathrm{s}$ $n^{\circ} 7$, vol. 2, p. 410). Ces résultats sont importants car ils montrent l'un des mécanismes par lesquels des protéines de type ElA peuvent entraîner la cancérisation cellulaire : en stimulant en trans l'expression de l'oncogène $m y c .$. dont on ne sait pas, à dire vrai, comment il agit lui-même! Cette action de ElA n'est d'ailleurs pas exclusive ; rappelons en effet que cette protéine peut aussi former un complexe stable avec le produit de l'anti-oncogène $\mathrm{Rb}\left(\mathrm{m} / \mathrm{s} n^{\circ} 8\right.$, vol. 4 , p. 520-521).

[1. Thalmeier K, et al. Genes Dev $1989 ; 3: 527-36$.]

[2. Hiebert SW, et al. Proc Natl Acad 514
Hyperexpression de l'oncogène HER-2/neu dans les cancers de l'ovaire... au même titre que dans les cancers du sein. L'oncogène $c$-erbB2 ou HER-2/neu est souvent amplifié dans les cancers mammaires et cette amplification, accompagnée d'une hyperexpression de la protéine, aurait, pour l'équipe de D. J. Slamon (UCLA school of medicine, Los Angeles, $\mathrm{Ca}$, USA), une très importante valeur pronostique $(\mathrm{m} / \mathrm{s}$ $n^{\circ} 2$, vol. 5 , p. 121). Outre son amplification, le gène $H E R-2 / n e u$ détecté dans les tumeurs mammaires a subi une mutation ponctuelle siégeant dans la région transmembranaire de la protéine Neu, protéine qui a toutes les caractéristiques d'un récepteur hormonal ou d'un facteur de croissance et qui possède un domaine intracytoplasmique de type «tyrosine-kinase». La mutation remplace une valine par une isoleucine et on ne sait pas si elle joue un rôle dans le pouvoir oncogénique de cette protéine Neu [1]. Chez le rat, en revanche, il a été démontré qu'une mutation valine $\rightarrow$ acide glutamique dans cette même région transmembranaire était capable, par ellemême, de rendre oncogénique le gène $H E R$-neu. Les protéines Neu ainsi modifiées tendent à s'agréger sur la membrane cellulaire, ce qui mime la réaction de récepteurs du type de ceux pour l'insuline et pour EGF (epidermal growth factor) lorsqu'ils lient leur ligand [2]. Dans sa récente étude menée en collaboration avec six autres laboratoires, D.J. Slamon confirme et étend ses résultats antérieurs sur la valeur pronostique d'une hyperexpression du gène $H E R-2 / n e u$, analysée au niveau du nombre de copies du gène, de l'abondance des ARNm ou de celle de la protéine [1]. Il établit aussi que ce même phénomène pourrait être observé dans le cancer de l'ovaire, cancer qui a de nombreux points communs avec celui du sein, notamment la fréquente expression de récepteur pour les œestrogènes par les cellules tumorales. Après maints débats, ces résultats semblent établir que l'oncogène $H E R-2 / n e u$ joue un rôle crucial dans la carcinogenèse mammaire et peut-être ovarienne. C'est à un même résultat qu'aboutissait l'analyse des souris transgéniques exprimant un oncogène $H E R$ neu «activé »: elles développaient constamment des tumeurs mammaires massives, polyclonales $(\mathrm{m} / \mathrm{s}$ $n^{\circ} 2$, vol. 5, p. 121)

[1. Slamon DJ, et al. Science 1989 ; 244 : 707-12.]

[2. Weiner DB, et al. Nature 1989; 339 : 230-1.]

Une myopathie mitochondriale avec délétions multiples de l'ADN mitochondrial est transmise comme un caractère autosomique dominant. Des équipes italienne et américaine viennent de décrire une très intrigante observation de myopathie mitochondriale typique (ophtalmoplégie externe bilatérale, faiblesse des muscles proximaux, cataracte bilatérale et décès précoce) transmise sur un mode autosomique dans une large famille italienne [1]. Chez quatre malades, des délétions variables sont retrouvées au niveau de l'ADN mitochondrial; des molécules d'ADN mitochondrial intactes coexistent avec des molécules ayant subi des délétions (phénomène d'hétéroplasmie). Toutes les délétions débutent dans une séquence de 12 nucléotides qui est très conservée dans plusieurs espèces de mammifères et qui joue un rôle important dans la réplication du génome mitochondrial et dans sa transcription (région distale de la boucle de déplacement, ou boucle $\mathrm{D}$, au niveau de laquelle débute la réplication du brin léger de la molécule d'ADN mitochondrial). A ce niveau, des interactions complexes entre l'ADN et des protéines intervenant dans la réplication et/ou dans la transcription se produisent ; il est certain que la majorité ou la totalité de ces pro- 
téines est codée par des gènes nucléaires. Une anomalie de l'une de ces protéines (topo-isomérase, primase, polymérase, déroulase, etc.) pourrait entraîner des modifications de la structure de l'ADN en cours de réplication, à l'origine de délétions qui seraient donc des phénomènes secondaires, aléatoires, expliquant l'hétérogénéité des remaniements observés.

[1. Zeviani M, et al. Nature 1989 ; 339 : 309-11.]

\section{Dolymorphisme génétique du} gène de la rénine chez le rat $\mathrm{Dahl}$. Les rats sélectionnés par L.K. Dahl sont subdivisés en deux groupes : les rats sensibles (S) qui deviennent hypertendus sous administration de sel, et les rats résistants ( $R$ ) qui restent normotendus malgré le sel. Le groupe de J.P. Rapp a étudié dans ces deux souches le gène de la rénine à l'aide de 28 enzymes de restriction: huit polymorphismes entre les deux souches $\mathrm{S}$ et $\mathrm{R}$ ont été mis en évidence [1]. Les gènes de la rénine chez $\mathrm{S}$ et $\mathrm{R}$ ont été clonés et de multiples différences ont été observées : notamment une insertion/délétion de $1,2 \mathrm{~kb}$ dans le premier intron $\mathrm{du}$ gène [2]. A partir des deux souches $S$ et $\mathrm{R}$, des hybrides $\mathrm{Fl}$ puis $\mathrm{F} 2(\mathrm{Fl} X$ Fl) ont été produits. La pression artérielle est très hétérogène chez les rats F2 soumis à un régime riche en sel ; cependant le niveau de la pression artérielle dépend étroitement de la présence ou non de l'allèle rénine $\mathrm{S}$ dans le génome; chez les rats F2 homozygotes pour cet allèle, la pression artérielle s'élève de $20 \mathrm{mmHg}$ environ alors qu'une seule dose de cet allèle entraîne une augmentation de $10 \mathrm{mmHg}$ [3]. Comme les rats Dahl S ont une rénine basse, ces résultats suggèrent que l'allèle $S$ est probablement responsable de cet abaissement et co-ségrège avec une augmentation de la pression artérielle. Le déterminisme de la pression artérielle est polygénique ; les résultats de Rapp et al. montrent qu'un gène impliqué dans la régulation de $m / s n^{\circ} 7$ vol. 5 , septembre 89 la pression artérielle est localisé dans le gène de la rénine ou dans une région du génome qui lui est proche. [1. Dene $\mathrm{H}$, et al. J Hypertension $1989 ; 7: 121-6$.]

[2. Wang SM, Rapp JP. Mol Endocrinol 1989 ; 3 : 288-94.]

[3. Rapp JP, et al. Science $1989 ; 243$ : 542-4.]

Les cyclodextrines, glucides de synthèse capables de contrôler l'angiogenèse. L'angiogenèse joue un rôle essentiel dans l'évolution de nombreuses maladies et en particulier des tumeurs (voir m/s $n^{\circ} 5$, vol. 4, p. 318). Il serait donc très important de s'en assurer la maîtrise pour en moduler le rythme, en l'accélérant ou en la ralentissant. A l'heure actuelle, il est possible de l'inhiber en combinant l'action de l'héparine ou d'un de ses fragments avec celle de stéroïdes dits angiostatiques [1], alors que l'héparine seule l'active et que les stéroïdes seuls sont sans effets. L'hétérogénéité des préparations d'héparine a fait souhaiter leur remplacement par des composés mieux définis. Folkman et al. (Harvard et Philadelphie) proposent [2] d'utiliser les cyclodextrines (CD), qui sont des composés cycliques solubles, non réducteurs (comme l'inositol), formés de six à huit cycles hexagonaux d'hexose. Leur molécule laisse à l'intérieur une cavité hydrophobe, dont le diamètre est déterminé par le nombre des unités hexose (six, sept ou huit pour les $\operatorname{CD} \alpha, \beta$ et $\gamma$ respectivement). A cette cavité peuvent s'adapter notamment des stéroïdes. Les groupes hydroxyles localisés à la surface peuvent être remplacés par des ligands destinés à augmenter l'hydrophilie et le pouvoir de liaison de la molécule. De ces substituants, le plus actif est le sulfate. Le meilleur composé synthétisé est un tétradécasulfate de $\beta C D$, complexé à l'hydrocortisone ou à un de ses dérivés peu actif métaboliquement, la cortexolone* ${ }^{*}$. Utilisant le test in vitro de la membrane chorio-allantoïque du poulet, on obtient une activité anti- angiogénique très supérieure à celle des héparines commerciales. Dans le test in vivo de la cornée du lapin, l'effet d'une implantation d'endotoxine est contrecarré par un implant de $\beta C D$-tétrasulfate-cortexolone, mais aussi par l'instillation de gouttes sur la cornée. Les auteurs proposent un modèle où le glucide apporte le stéroïde à la surface de la cellule endothéliale à laquelle il l'amarre par des liaisons hydrophiles solides. Le $\beta$ cyclodextrine tétrasulfate est donc un inhibiteur potentiel de l'angiogenèse lorsqu'il est administré combiné à un composé angiostatique comme certains stéroïdes ; il pourrait, réciproquement, promouvoir l'angiogenèse si on le liait à des molécules angiogéniques.

[1. Folkman J, et al. Science 1983 ; 221 : 719-25.]

[2. Folkman J, et al. Science 1989; 243 : 1490-3.]

* La cortexolone dérive de l'hydrocortisone par perte du groupement $\mathrm{OH}$ en position 11.

Le neurolathyrisme continue à sévir en Inde. Une «brève » parue dans $\mathrm{m} / \mathrm{s}$ en 1987 ( $\mathrm{n}^{\circ} 3$, vol. 3, p. 116) mentionnait que les signes neurologiques graves du lathyrisme pouvaient être attribués à un dérivé toxique d'acide aminé, la $\beta-\mathbf{N}$ oxalylaminoalanine, contenu dans la plante Lathyrus sativus (gesse chiche). Nous pensions cependant qu'il s'agissait là d'une intoxication « historique». Il semble malheureusement qu'il n'en soit rien. D'après Jayaraman [1], dans ce même état de Madyha Pradesh qui a connu le drame de Bhopal, un nombre encore plus élevé de victimes, et qui elles n'ont aucun espoir de dédommagement, continuent à consommer cette plante qui ne réclame ni irrigation ni engrais. Les paysans reçoivent des aliments contenant du Lathyrus sativus de leurs propriétaires à titre de salaire. Une lueur d'espoir pourtant : la baisse du prix du riz et du blé devrait permettre l'abandon progressif de la plante toxique.

[1. Jayaraman KS. Nature 1989, 339 : 495.]

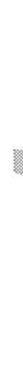

